\title{
The effect of complicated febrile convulsion on hippocampal function and its antiepileptic treatment significance
}

\author{
Bin Li, Youjia Wu, Qingjuan He, Hui Zhou, Jin Cai \\ Department of Pediatrics, Affiliated Hospital of Nantong University, Nantong, China \\ Contributions: (I) Conception and design: B Li, J Cai; (II) Administrative support: None; (III) Provision of study materials or patients: None; (IV) \\ Collection and assembly of data: All authors; (V) Data analysis and interpretation: All authors; (VI) Manuscript writing: All authors; (VII) Final \\ approval of manuscript: All authors. \\ Correspondence to: Jin Cai; Hui Zhou. Department of Pediatrics, Affiliated Hospital of Nantong University, Nantong 226001, China. \\ Email: Sunnyvip2020@163.com; nthuihui@126.com.
}

Background: This study aimed to explore the effects of complex febrile seizures on hippocampal function and the significance of antiepileptic therapy.

Methods: A total of 150 children with complex febrile seizures admitted to our hospital from July 2017 to July 2020 were included in the study. The VPA group was given sodium valproate treatment; the LEV group was given levetiracetam treatment; and the observation group was given basic treatment. The efficacy of the patients was evaluated after medication. A complex febrile seizure young mouse model was constructed, and the hippocampal cell morphology and BCL-2 expression of the mice pups were analyzed. A Morris water maze was used to detect the changes in cognitive function of the young mice with complex febrile seizures.

Results: After treatment, the recurrence-free rate of the VPA group was significantly higher than that of the observation group ( $\mathrm{P}=0.0045)$. After 1 month and 6 months, the improvement rate of EEG in VPA group was significantly higher than that in observation group $(\mathrm{P}<0.05)$. After treatment, the levels of BCL-2 in the VPA group and the LEV group decreased and were significantly lower than the observation group during the same period $(\mathrm{P}<0.05)$, and the $\mathrm{M} / \mathrm{C}$ of the two groups was significantly higher than the observation group $(\mathrm{P}<0.05)$. The neuronal cells in the hippocampus of the young rats in the VPA group and the LEV group were regular, the matrix was more uniform, and nuclear pyknotic cells were occasionally seen. The pathological changes were less obvious than the model group, followed by the degree of pathological changes $(0.92 \pm 1.31,0.94 \pm 1.24)$. The incubation period of pups in the model group was significantly higher than that of the normal group, VPA group, and LEV group $(\mathrm{P}<0.05)$, and the number of crossing the station area was significantly less than that of the normal group, VPA group, and LEV group $(\mathrm{P}<0.05)$.

Conclusions: Antiepileptic drugs are effective in preventing the recurrence of complicated febrile seizures (CFS), and the main mechanism may be related to the targeted regulation of BCL-2 on the apoptosis of the hippocampus in the nervous system.

Keywords: Complex febrile convulsions; hippocampal function; antiepileptic drugs; BCL-2

Submitted Nov 24, 2020. Accepted for publication Jan 26, 2021.

doi: $10.21037 / \mathrm{tp}-20-458$

View this article at: http://dx.doi.org/10.21037/tp-20-458

\section{Introduction}

Febrile seizures are one of the most common types of seizures in children, and usually occur between the ages of 6 months and 5 years (1). Complex febrile convulsions account for $20 \%$ of febrile convulsions, and the prevalence among children in China is $3.9 \%$. Complex febrile convulsions are characterized by localized seizures (confined to a single limb or unilateral body axis) which last $>15$ minutes or recur within 24 hours (2). Although complex febrile seizures have 
a good prognosis, they can cause varying degrees of brain damage, related histopathological changes, and adversely affect long-term learning and memory.

A small sample study found that a short time after the onset of prolonged febrile seizures, cognition, facial recognition, and memory were impaired, and this was found to be related to impairment of the cognitive function of the hippocampus (3). However, there is currently a lack of large sample-sized research of nervous system damage for complex febrile seizures in China and abroad. In addition to the impairment of hippocampal cognitive function, the incidence of epilepsy in the history of complicated febrile seizures (CFS) (2-3\%, 6-50\%, respectively) has been significantly increased. These figures highlight that CFS are not an absolutely benign disease. Selective drug therapy is necessary to prevent ongoing negative outcomes. In recent years, some researchers have shown that antiepileptic drugs are beneficial to the clinical remission of patients in the treatment of complex febrile convulsions $(4,5)$.

The hippocampus is an important area of the brain for high-level neural activity, learning, and cognition. The $B C L-2$ gene encodes a $26-\mathrm{kDa}$ integral membrane protein located in the outer mitochondrial membrane and endoplasmic reticulum. Studies have found that BCL-2 is involved in the regulation of cognitive memory in the hippocampus (6). In mouse model experiments, increasing the level of BCL-2 improved the cognitive function of the hippocampus (7). Complex febrile seizures and BCL-2 are closely related to the hippocampus. The abovementioned studies suggest that BCL-2 may play a certain role in the process of complex febrile seizures in children, and its underlying mechanism may be related to the targeted regulation of the apoptosis of hippocampal tissues in the nervous system. Based on this, we included cases of complex febrile convulsions and explored their impact on hippocampal function and the significance of anti-epileptic therapy in clinical treatment. The proposed application of anti-epileptic drugs was complicated due to risk factors for recurrence and possible later secondary epilepsy. Children with febrile seizures were treated with drugs to prevent the recurrence of complex febrile seizures. Adverse reactions, EEG, hippocampal lesion improvement, and BCL-2 levels were analyzed after administration of the antiepileptic drugs. At the same time, because pathological tissues of child participants could not be obtained, and the EEG of febrile seizure mice is similar to that of human febrile activity (8), we established a corresponding mouse model to obtain the pathological tissue of the mouse. We were then able to perform in-depth explorations to ascertain the pathogenesis of complex febrile seizures and verify whether BCL-2 can be used as an early warning indicator.

We present the following article in accordance with the MDAR reporting checklist (available at http://dx.doi. org/10.21037/tp-20-458).

\section{Methods}

\section{Source of cases}

A total of 150 children with first-onset of CFS who were hospitalized or treated as outpatients in the Pediatrics Department of Nantong University Hospital from July 2017 to July 2020 were selected for this study. Inclusion criteria were as follows: (I) main criteria: (i) partial seizures; (ii) long-term seizures ( $\geq 15 \mathrm{~min}$ ); (iii) $\geq 3$ cluster seizures within 24 hours; (iv) neurological abnormalities after the attacks; (v) repeated attacks, the total number of times was at least 5. (II) Secondary criteria: (i) the age of first episode was $<18$ months; (ii) an abnormal development of the nervous system; (iii) a family history of epilepsy or a history of febrile seizures (FS) in a first-degree relative; (iv) the time from onset of fever to convulsion $<1$ hour. Children who met any 3 of the main criteria, or any 2 plus any 1 of the minor criteria were deemed to have met the inclusion criteria. (III) The exclusion criteria were as follows: (i) central nervous system infection; (ii) previous febrile convulsions; (iii) severe metabolic and electrolyte imbalances.

In addition, 50 healthy children were included as a control group, and the results of the Webster's Intelligence Scale, EEG, and MRI were collected. All procedures performed in this study involving human participants were in accordance with the Declaration of Helsinki (as revised in 2013). The study was approved by Affiliated Hospital of Nantong University (No. 2019005) and informed consent was taken from all the patients.

\section{Treatment plan and efficacy evaluation}

A total of 54 patients in the VPA group were given sodium valproate. There were three dosage forms of sodium valproate, namely sodium valproate oral solution (trade name Debajin) $300 \mathrm{~mL} /$ bottle, sodium valproate sustainedrelease tablets (trade name Debajin) $0.5 \mathrm{~g} /$ tablet (30 tablets), and $\mathrm{C}$ magnesium valproate sustained-release tablets $0.25 \mathrm{~g} /$ tablet (30 tablets); the initial oral dose was $10 \mathrm{mg} / \mathrm{kg} / \mathrm{d}$, and the final dosage was $20-40 \mathrm{mg} / \mathrm{kg} / \mathrm{d}$. 
After 5 half-lives time of the medication had elapsed, we checked the blood concentration of sodium valproate and ensured it was maintained at $50-100 \mu \mathrm{g} / \mathrm{mL}$. A total of 52 cases in LEV group were given levetiracetam. There were two dosage forms of levetiracetam, namely levetiracetam oral liquid (trade name Kaipulan) $150 \mathrm{~mL}$ (1 bottle) and levetiracetam tablets (trade name Kaipulan) $0.25 \mathrm{~g} /$ tablet (30 tablets); the initial oral dose was $10 \mathrm{mg} / \mathrm{kg} / \mathrm{d}$, and the maintenance dose was $20-40 \mathrm{mg} / \mathrm{kg} / \mathrm{d}$. A total of 42 cases were included in the observation group, after exclusion of participants whose family members did not consent to them receiving anti-epileptic treatment, and basic treatment was given to these remaining participants.

Divisions were made according to whether there was recurrence after taking the drug: (I) No recurrence: the seizure was completely controlled after the administered drug had reached the basic dose, and there was no recurrence, (II) recurrence: the condition was completely controlled after the maximum tolerated dose had been reached, but then another attack occurred. Within the recurrence group, there were seizures, but according to the degree of reduction in the number of seizures, the following categories were formed: (I) a reduction of $\geq 75 \%$ was deemed markedly effective; (II) a reduction of $\geq 50 \%$ was deemed effective; (III) a reduction of $<50 \%$ was deemed ineffective; (IV) exacerbation: (i) the number of seizures increased compared with before medication; (ii) afebrile convulsions appeared; (iii) convulsions persisted.

\section{EEG/MRI examination of the hippocampus}

We performed EEG and MRI examinations of the hippocampus within a week and one month after the onset of CFS in the child participants, and repeated the same examinations six months later. The scalp electrodes were installed using a 10-20 system for EEG detection. Single and bipolar tracing was performed using a bipolar 16lead. The EEG tracing time was no less than 20 minutes. Children $>3$ years old were subject to hyperventilation and eyes open and closed testing; if the child was uncooperative, $10 \%$ chloral hydrate was orally administered and then EEG was conducted as they slept. The examination results were deemed either normal or abnormal, and abnormalities included non-specific EEG (extensive slow waves) and paroxysmal abnormalities (spike waves, sharp waves, and spike (tip) slow comprehensive waves). The MR data acquisition was completed on a Siemens Skyra 1.5T superconducting magnetic resonance scanner (Siemens,
Munich, Germany). The sequence used was a T1-weighted three-dimensional magnetization preparation gradient echo sequence. The scanning parameters were as follows: repetition time $(\mathrm{TR})=2,300 \mathrm{~ms}$; echo time $(\mathrm{TE})=2.26 \mathrm{~ms}$; flip angle $(\mathrm{FA})=80$; acquisition matrix $=256 \times 200$; voxel size $=1 \mathrm{~mm} \times 1 \mathrm{~mm} \times 1 \mathrm{~mm}$; layer thickness $=1 \mathrm{~mm}$; layer distance $=0.5 \mathrm{~mm}$. The images obtained were used to assess hippocampal damage, whether there were changes in hippocampal volume and T2 signal, and whether hippocampal damage was unilateral or bilateral.

\section{Determination of serum BCL-2 levels in cases}

We collected $3 \mathrm{~mL}$ of venous peripheral blood from the case participants and healthy volunteers, centrifuged them at 3,000 r/min for 15 minutes to obtain serum, and then stored them at $-80{ }^{\circ} \mathrm{C}$. An enzyme-linked immunosorbent assay (ELISA) detection kit (PK-EL-69056, PromoCell, Heidelberg, Germany) was used to determine the level of BCL-2 in serum.

\section{Webster's Intelligence Scale}

The intelligence measurement was based on the Wechsler Intelligence Scale (9) formulated by Professor D. Wechsler (USA) and later revised by Professor Lin Chuanding and Zhang Houcan of the Beijing Normal University. After the measurement, the total words of the case participants and healthy volunteers were calculated, along with intelligence quotient (VIQ), operational intelligence quotient (PIQ), total intelligence quotient (FIQ), and memory/attention factor $(M / C)$.

\section{The establishment and grouping of complex febrile seizure models in mice}

A total of 15, 21-day-old pups were used to establish a complex animal model of febrile seizures. The specific method of establishment was as follows: experimental animals were treated with a hot water bath, and $10 \mathrm{~mL} / \mathrm{kg}$ of normal saline was injected subcutaneously before hyperthermia induction to avoid dehydration of the pups. The mice were then placed in a constant temperature water bath at $43.00 \pm$ $0.5^{\circ} \mathrm{C}$, and their heads were kept above the water. During the experiment, rectal temperature was measured every 2 minutes; 30 minutes after the onset of tonic convulsions, the animal was moved to a lower temperature surface until its basal body temperature was completely restored and it 
was then returned to the nest. After successfully establishing complex febrile seizures, the mice were divided equally into three groups. The LEV group was given the drug levetiracetam for 25 days, the VPA group was given the drug sodium valproate for 25 days, and the model group was administered normal saline for 25 days. In addition, 5 healthy pups were included as the normal group.

\section{Hematoxylin and eosin staining of mouse hippocampus and determination of $B C L-2$}

At the end of the experiment, the animals were sacrificed, and their brain tissues were placed in 10\% formalin for fixation, paraffin-embedded, sliced, and hematoxylin and eosin (HE) stained. Pathologists read the slices, and observed the hippocampus areas 1, 2, 3, and 4 (that is, CA1, CA2, CA3, and CA4) for whether the pyramidal cells in the area displayed degeneration, necrosis, alignment, and whether there were lesions indicative of inflammatory cell infiltration. The degree of the disease of each area was quantified as " 0 points" for normal, " 0.5 points" for a slight or very small amount, "1 point" for a mild or small amount, "2 points" for moderate or above, "3 points" for a severe or excessive amount, and " 4 points" for extremely severe or large amounts. All the scores were accumulated and the average score was calculated (mean \pm standard deviation) for each animal in each group. The higher the score, the more serious the injury.

After the brain tissue slices were roasted and dried, they were dewaxed and dehydrated, repaired by highpressure heat, and endogenous peroxidase was inactivated. The immunohistochemical (IHC) technique was used for staining. After successful staining, the sections were dried naturally, and mounted with neutral resin. Picture collection and picture analysis involved the observation of brain tissue slices under an optical microscope. The cytoplasm and envelope were brown when positively expressed; pictures were taken with high magnification $(40 \times 10)$ and ImageProPlus software was used to analyze and calculate the average optical density value $=\mathrm{IOD} /$ area $(\mathrm{AOD})$.

\section{Water maze test}

The experimental water tank was a galvanized round tank with a diameter of $150 \mathrm{~cm}$ and a height of $50 \mathrm{~cm}$; water depth was $25 \mathrm{~cm}$, water temperature was $22 \pm 2{ }^{\circ} \mathrm{C}$, and ink was added to the water to make the pool opaque. The pool was artificially divided into four quadrants, in a certain quadrant, a platform with a height of $23.5 \mathrm{~cm}$ and diameter of $8 \mathrm{~cm}$ was placed $25 \mathrm{~cm}$ from the edge of the pool, concealed $1.5 \mathrm{~cm}$ below the water surface. In the experiment, the pups were launched from the four quadrants at different entry points, but the quadrants were the same each time the pups entered the water. The pups were positioned facing the pool wall when entering the water. We recorded the time from entering the water to climbing onto the platform to indicate the latency of looking for the platform. If the baby rat did not find the platform within 120 seconds, the tester would guide it to the platform and repeat the test twice a day. After completing four positioning voyages in the morning of the eighth day, the platform was removed, the midpoint of the quadrant opposite the platform area was selected as the entry point, and the total number of times that the pups crossed the platform area to search for the platform within 120 seconds was recorded.

\section{Statistical methods}

The statistical software SPSS 21.0 (IBM, Chicago, IL, USA) was used to process the data in this study, the measurement data were expressed as mean \pm standard deviation, and the $t$-test was used for statistical analysis. The count data were expressed as rates, using the chi-squared $\left(\chi^{2}\right)$ test. A P value $<0.05$ was considered statistically significant.

\section{Results}

\section{The significance of anti-epileptic treatment of complex febrile convulsions}

\section{Baseline data}

There was no significant difference in the baseline data of the three groups of patients $(P>0.05)$. A week after the seizures had stopped, the EEG and MRI examinations of each group of children were performed. The detailed results are shown in Table 1.

\section{Outcome}

The recurrence-free rate of the VPA group was significantly higher than that of the observation group $\left(\chi^{2}=8.077\right.$, $\mathrm{P}=0.0045)$. Compared with the observation group, the recurrence-free rate of the LEV group was not statistically significant $\left(\chi^{2}=0.9469, \mathrm{P}=0.3350\right)$. The constituent ratios of the number of people with aggravated conditions in the VPA and LEV groups were significantly lower than those 
Table 1 Baseline data



VEP, sodium valproate; LEV, levetiracetam; EEG, electroencephalogram

in the observation group $\left(\chi^{2}=4.075, \mathrm{P}=0.0435 ; \chi^{2}=3.874\right.$, $\mathrm{P}=0.0490)$. A month later, the EEG improvement rate of the VPA group was significantly higher than that of the observation group $\left(\chi^{2}=8.077, \mathrm{P}=0.0045\right)$. Compared with the observation group, the EEG improvement rate of the LEV group was not statistically significant $\left.\left(\chi^{2}=0.6152\right), \mathrm{P}=0.4329\right)$ (Figure 1). After half a year, the improvement rate of hippocampal lesions in the VPA group was significantly higher than that in the observation group $\left(\chi^{2}=4.267\right.$, $\mathrm{P}=0.0389$ ). See Tables 2 and 3 for details.

\section{Changes in serum BCL-2 levels}

Before treatment, serum BCL-2 in the VPA, LEV, and observation groups were significantly higher than those in the healthy control group $(\mathrm{P}<0.05)$. After treatment, the level of BCL-2 in the VPA and the LEV groups decreased, and was significantly lower than the observation group during the same period $(\mathrm{P}<0.05)$ (Table 4).

\section{Determination of Webster's Intelligence Scale}

Compared with the healthy control group, the VIQ, PIQ, and FIQ of the patients in the VPA, LEV, and observation groups before and after treatment were not statistically significant $(\mathrm{P}>0.05)$. Before treatment, the $\mathrm{M} / \mathrm{C}$ of the three participant groups was significantly lower than that of the healthy control group $(\mathrm{P}<0.05)$. After treatment, the $\mathrm{M} / \mathrm{C}$ of the VPA and LEV groups was significantly higher than that of the observation group $(\mathrm{P}<0.05)($ Table 5$)$.

\section{Adverse reactions}

The incidence of adverse reactions in the VPA group was $5.56 \%(3 / 54)$, and the incidence of adverse reactions in the LEV group was $26.92 \%$ (14/52). There was a statistical difference between the two groups $\left(\chi^{2}=8.982, \mathrm{P}=0.0027\right)$. Among them, in the VPA group, 1 case had hair loss, 1 case had weight gain, and 1 case had a slight decrease in platelets. In the LEV group, 4 cases developed dizziness and vomiting, 4 cases developed irritability and short temper, 3 cases developed hyperactivity and lack of concentration, and 3 cases experienced loss of appetite. Both groups of study participants were closely monitored and followed up, and none of them stopped the drug due to intolerable adverse reactions.

\section{The role of BCL-2 in hippocampal dysfunction in young rats with complex febrile seizures}

The pathological manifestations of hippocampal nerve cells in a young mouse model of complex febrile seizures As can be seen in the Figure $2 A$, the hippocampal pyramidal 


Table 2 Disease outcome

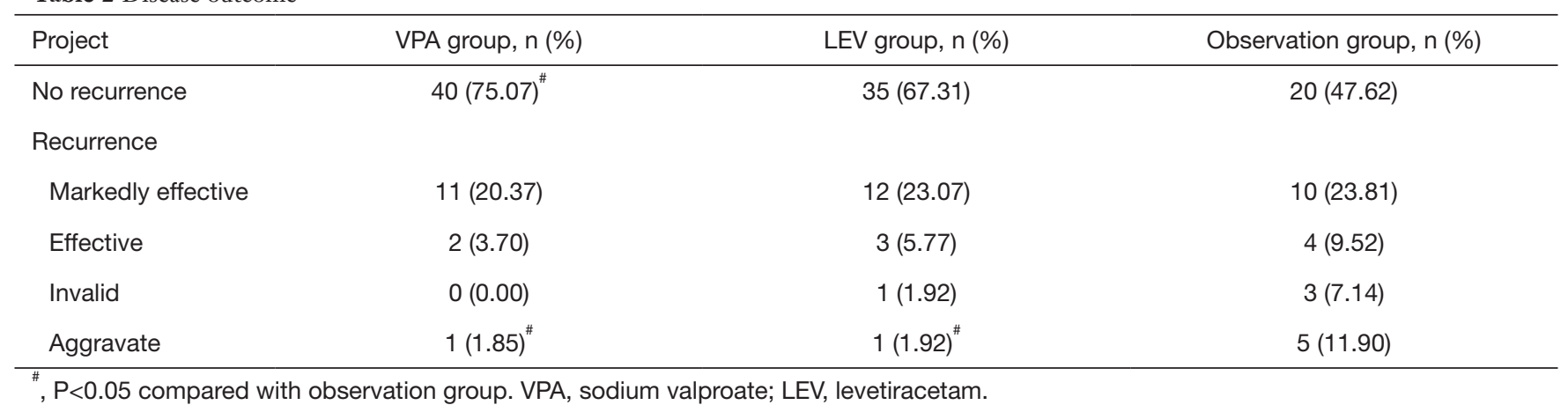

Table 3 Results of EEG/hippocampal MRI

\begin{tabular}{|c|c|c|c|c|c|c|}
\hline Project & \multicolumn{2}{|c|}{ VPA group, n (\%) } & \multicolumn{2}{|c|}{ LEV group, $\mathrm{n}(\%)$} & \multicolumn{2}{|c|}{ Observation group, n (\%) } \\
\hline \multicolumn{7}{|l|}{ EEG } \\
\hline Improve & $18(85.71)^{\#}$ & $20(95.24)$ & $16(72.72)$ & $18(81.82)$ & $11(64.71)$ & $14(82.35)$ \\
\hline No change & $3(14.29)$ & $1(4.76)$ & $5(22.73)$ & $4(18.18)$ & $4(23.53)$ & $3(17.65)$ \\
\hline \multicolumn{7}{|l|}{ MRI } \\
\hline Improve & $3(37.50)$ & $5(62.50)^{\#}$ & $2(25.00)$ & $4(50.00)$ & $1(12.50)$ & $1(12.50)$ \\
\hline No change & $5(62.50)$ & $3(37.50)$ & $6(75.00)$ & $4(50.00)$ & $5(62.50)$ & $6(75.00)$ \\
\hline Aggravate & $0(0.00)$ & $0(0.00)$ & $0(0.00)$ & $0(0.00)$ & $2(25.00)$ & $1(12.50)$ \\
\hline
\end{tabular}

cells of the normal group of young rats are neatly arranged, with clear layers, the pyramidal cell nucleus is large and round, there are obvious nucleoli, and a large number of cells. The pyramidal cells of the young rats in the model group were degenerated, with mild, moderate, or very severe nuclear pyknosis; the neuronal nuclei were swollen (Figure 2B). The neurons in the hippocampus of the VPA and LEV groups were regular and the matrix was relatively uniform. There were occasional nuclear pyknotic cells, and the pathological changes were unknown (Figure 2C,D). To evaluate the degree of lesions in different groups of mice, the lesion score of the model group $(2.83 \pm 2.84)$ was significantly higher than that of the normal group $(0.83 \pm 1.33)$, VPA group $(0.92 \pm 1.31)$, and LEV group $(0.94 \pm 1.24)(\mathrm{P}<0.05)$.

\section{Determination of BCL-2 in young rats with complex febrile seizures}

The presence of BCL-2 positive cells in the hippocampus of pups in the normal group was rare (Figure $3 A$ ); there were more BCL-2 positive cells in the hippocampus of pups in the model group, and the coloring of the positive cells and the average optical density were enhanced (Figure 3B). The BCL-2 positive cells in the hippocampus of young rats in the VPA and LEV groups decreased, and the coloring and average optical density of the positive cells decreased (Figure 3C,D). Comparing the average optical density values of pups in different groups, the average optical density of BCL-2 in the hippocampus of young rats in the model group $(129.52 \pm 7.25)$ was significantly higher than that in the normal $(120.60 \pm 6.39)$, VPA $(121.03 \pm 6.27)$, and LEV groups (120.88 \pm 6.14$)$.

Morris water maze test to detect the cognitive function changes of young mice with complex febrile seizures The incubation period of pups in the model group was significantly higher than that of the normal, VPA, and LEV groups $(\mathrm{P}<0.05)$, and the number of crossing the station area was significantly less than that of the normal, VPA, and 
LEV groups $(\mathrm{P}<0.05)$ (Table 6).

\section{Discussion}

Febrile convulsions often occur in childhood and are prone

Table 4 Changes of BCL-2 in serum

\begin{tabular}{|c|c|}
\hline Group & BCL-2 (ng/mL) \\
\hline \multicolumn{2}{|l|}{ VPA group } \\
\hline Before therapy & $6.67 \pm 1.29^{\Delta}$ \\
\hline After 1 month of treatment & $5.42 \pm 1.45^{\#}$ \\
\hline After 6 months of treatment & $4.96 \pm 1.78^{\#}$ \\
\hline \multicolumn{2}{|l|}{ LEV group } \\
\hline Before therapy & $6.48 \pm 1.34^{\Delta}$ \\
\hline After 1 month of treatment & $5.61 \pm 1.43^{\#}$ \\
\hline After 6 months of treatment & $5.09 \pm 1.78$ \\
\hline \multicolumn{2}{|l|}{ Observation group } \\
\hline Before therapy & $6.75 \pm 1.63^{\Delta}$ \\
\hline After 1 month of treatment & $6.42 \pm 1.54^{\#}$ \\
\hline After 6 months of treatment & $6.35 \pm 1.69^{\#}$ \\
\hline Healthy control group & $4.71 \pm 1.23^{\Delta}$ \\
\hline
\end{tabular}

to recurrence. A cohort study found that children should be evaluated immediately after the initial episode. The risk of recurrence is $15 \%$ to $70 \%$ at 2 years after the initial febrile seizure (10). The risk of recurrence is increased for patients $<18$ months, those with low fever, short duration of pre-onset fever, or with a family history of high fever episodes (11). Febrile seizures are divided into simple and complex types. Diagnosis should include distinction between the two types of patients, because complex febrile seizures can cause the destruction of nerve cells and impact upon the developing brain. I has been found that are the main risk factor for epilepsy; the probability of CFS transforming into epilepsy is 3.6 times that of simple febrile seizures, and this causes a heavy burden on individuals, families, and society (12). Children who experience febrile seizures have an increased risk of recurrence and nonfebrile seizures, which suggests that long-term use of antiepileptic drugs for preventive treatment may be useful. However, considering that recurrent febrile convulsions are benign, the risk assessment of antiepileptic drug therapy is still controversial $(13,14)$. The clinical practice guidelines developed by the Quality Improvement Committee of the American Academy of Pediatrics Febrile Convulsions propose that, based on the risks and benefits of effective treatment, potential epileptic syndromes for patients with CFS may be discovered by collecting a detailed clinical history and reviewing their EEG. After evaluation of the characteristics of anti-epileptic drugs, the benefits of such medications may outweigh the risks, and have a certain

Table 5 IQ test results

\begin{tabular}{|c|c|c|c|c|}
\hline Quotient project & VIQ & $\mathrm{PIQ}$ & $\mathrm{FIQ}$ & $\mathrm{M} / \mathrm{C}$ \\
\hline \multicolumn{5}{|l|}{ VPA group } \\
\hline Before therapy & $113.67 \pm 12.97$ & $102.85 \pm 13.69$ & $109.49 \pm 14.71$ & $30.74 \pm 6.03^{\Delta}$ \\
\hline After treatment & $114.52 \pm 13.58$ & $102.41 \pm 13.15$ & $110.23 \pm 15.26$ & $35.82 \pm 6.21^{\#}$ \\
\hline \multicolumn{5}{|l|}{ LEV group } \\
\hline After treatment & $112.95 \pm 12.87$ & $103.38 \pm 12.74$ & $109.36 \pm 15.39$ & $34.78 \pm 6.27^{\#}$ \\
\hline \multicolumn{5}{|l|}{ Observation group } \\
\hline Before therapy & $113.14 \pm 12.86$ & $103.27 \pm 13.56$ & $109.23 \pm 14.95$ & $31.12 \pm 6.27^{\Delta}$ \\
\hline After treatment & $114.12 \pm 13.45$ & $103.62 \pm 13.71$ & $109.84 \pm 15.17$ & $30.48 \pm 6.83$ \\
\hline
\end{tabular}

", $\mathrm{P}<0.05$ compared with observation group; ${ }^{\Delta}, \mathrm{P}<0.05$ compared with healthy control group. PIQ, operational intelligence quotient; FIQ total intelligence quotient; M/C memory/attention factor; VIQ/IQ, intelligence quotient; VPA, sodium valproate; LEV, levetiracetam. 

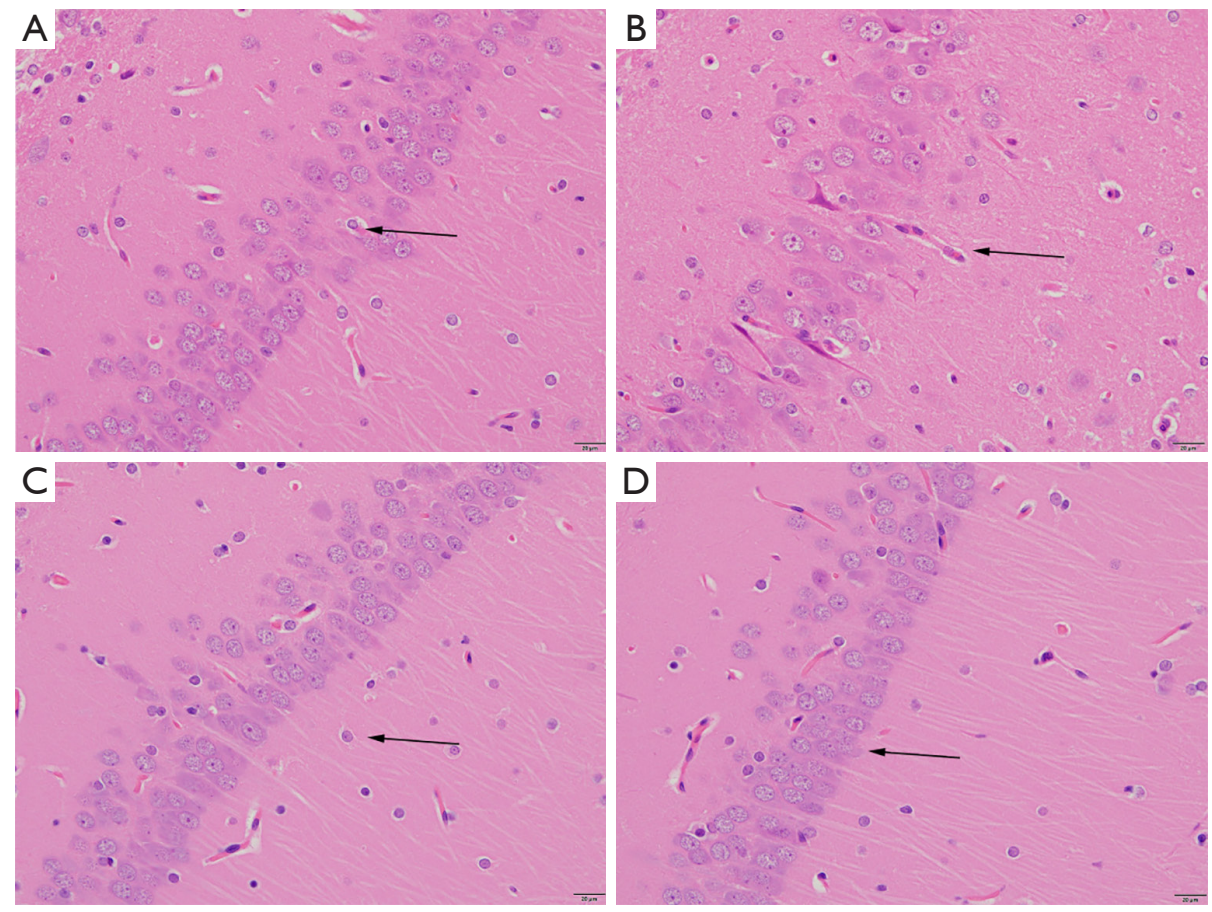

Figure 2 The morphological manifestations of nerve cells in the hippocampus of a young mouse model of complex febrile seizures (HE stain). (A) Normal group; (B) model group; (C) VPA group; (D) LEV group. Arrows mean the observation positions. VPA, sodium valproate; LEV, levetiracetam.
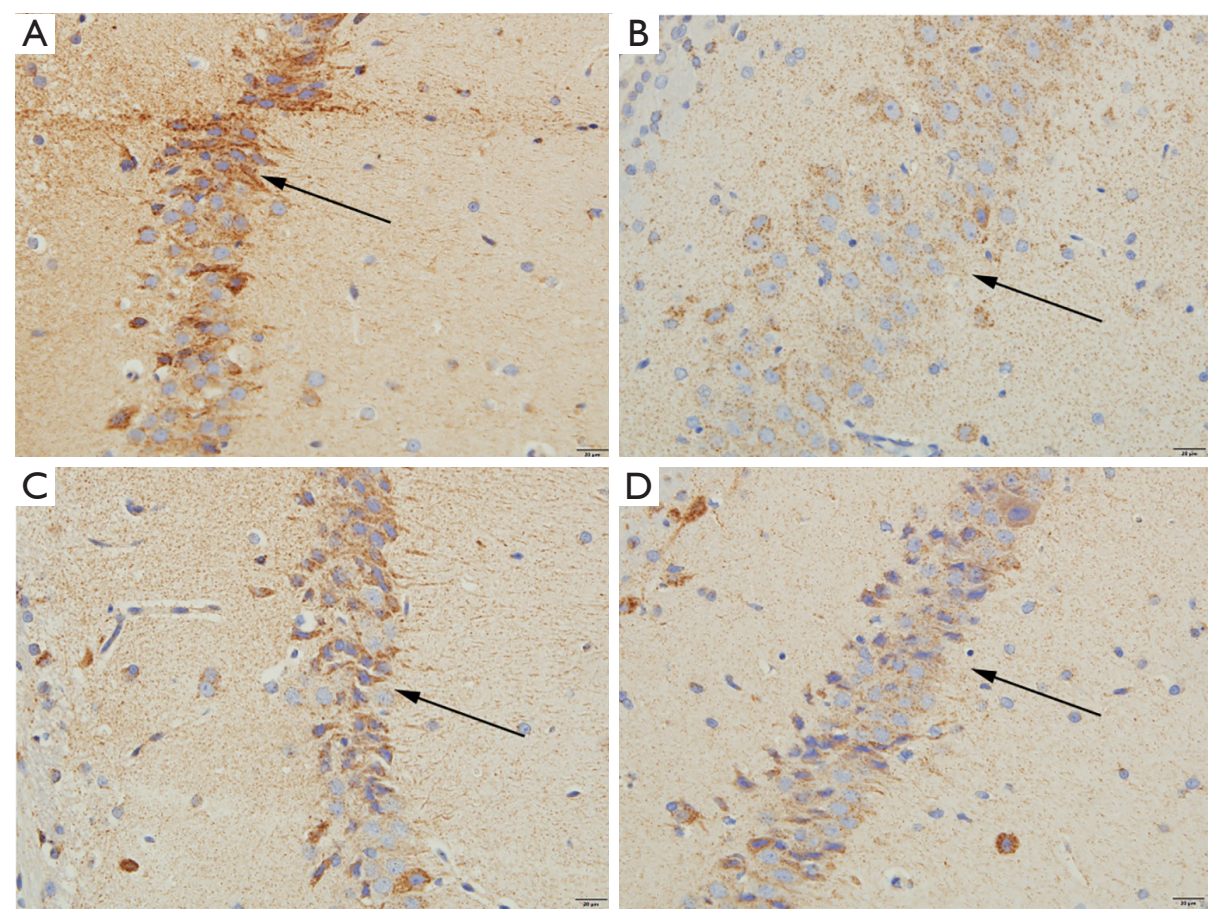

Figure 3 Expression of BCL-2 in the hippocampus of young rats in different groups (immunohistochemical staining). (A) Normal group; (B) model group; (C) VPA group; (D) LEV group. Arrows mean the observation positions. VPA, sodium valproate; LEV, levetiracetam. 
Table 6 Morris water maze test results

\begin{tabular}{lcc}
\hline Group & Incubation period & Frequency \\
\hline Model group & $20.89 \pm 7.61$ & $5.42 \pm 1.73$ \\
Normal group & $13.05 \pm 6.49^{\mathrm{a}}$ & $10.56 \pm 2.32^{\mathrm{a}}$ \\
VPA group & $13.78 \pm 6.83^{\mathrm{a}}$ & $10.09 \pm 2.18^{\mathrm{a}}$ \\
LEV group & $13.31 \pm 5.96^{\mathrm{a}}$ & $9.86 \pm 2.03^{\mathrm{a}}$ \\
\hline${ }^{\mathrm{a}}$, P<0.05 compared with the model group. VPA, sodium \\
valproate; LEV, levetiracetam.
\end{tabular}

effect on improving the prognosis. This is especially true in cases where parents are deeply concerned about the recurrence of convulsions and have carefully weighed up the risks of anti-epileptic drugs. The current commonly used medication regimen is continuous and uninterrupted long-term preventive medication, antiepileptic drugs such as VPA are used for long-term prevention, and the course of treatment is generally 2 years; that is, the medication is taken continuously until there are no further convulsions for $>2$ years, then the dose is slowly reduced, and finally stopped. It is mainly used in children with frequently occurring CFS or who have experienced a poor effect from short-term prevention methods. The clinical application of VPA to prevent the recurrence of CFS and the development of epilepsy has a history of $>20$ years. A meta-analysis by Rantala et al. (15) included 9 clinical studies to evaluate the efficacy of antiepileptic drugs in preventing the recurrence of febrile seizures. The results showed that compared with the placebo group, children taking sodium valproate [odds ratio $(\mathrm{OR})=0.090,95 \%$ confidence interval $(\mathrm{CI}): 0.010$ $0.780 ; \mathrm{P}=0.011$ ] had a significantly lower recurrence rate of febrile seizures.

In recent years, animal experiments and clinical studies on preventive medication with levetiracetam in complex febrile seizures have confirmed that the levetiracetam was highly effective in preventing CFS recurrence and have fewer adverse reactions (16). However, these study cohorts are not comparable due to differences in race, inclusion criteria, drug use, and criteria for disease outcome. At present, levetiracetam and sodium valproate are used to promote the effectiveness of the preventive treatment of complex febrile convulsions, improve abnormal EEG, and as post-medication therapy. Relatively few studies have addressed the comparative situation. The results of the clinical trials of this study were similar to those of the abovementioned studies. The recurrence-free rate of the VPA group was significantly higher than that of the observation group, but the recurrence-free rate of the LEV group was not significantly different from that of the observation group. The constituent ratios of the number of people with aggravated conditions in the VPA group and LEV group were significantly lower than those in the observation group, suggesting that sodium valproate and levetiracetam can prevent CFS to a certain extent. Sodium valproate is more effective than levetiracetam; analysis of the EEG improvement rates in each group showed a significant improvement in the VPA group after a month of treatment. The improvement rate of hippocampal lesions after half a year of treatment with VPA was significantly higher than that of the observation group, further confirming the efficacy of sodium valproate. Comparison of the incidence of adverse reactions in the VPA and LEV groups showed that sodium valproate had fewer adverse reactions than levetiracetam, which suggested that sodium valproate is the safer option.

The apoptosis pathway mediated by endoplasmic reticulum stress response will become one of the important areas of research in the field of convulsive brain injury, and BCL-2 plays an important role in endoplasmic reticulum stress-responsive apoptosis. Currently, there has been little research conducted to investigate whether the BCL-2 reactive apoptotic pathway is also involved in convulsive brain injury of the immature brain. In this study, comparison of the levels of BCL-2 before and after treatment, showed that before treatment, the serum BCL-2 in the VPA group, LEV group, and observation group were significantly higher than those in the healthy control group $(\mathrm{P}<0.05)$. After treatment, the level of BCL-2 in the VPA group and the LEV group had decreased, and was significantly lower than the observation group during the same period. Combined with the analysis of the results of the Webster's Intelligence Scale, the M/C of the three groups of participants before treatment was significantly lower than that of the healthy control group. After treatment, the M/Cs of the VPA and LEV groups were significantly improved, suggesting CFSs have an impact on the cognitive level of some individuals, and antiepileptic drugs can ameliorate this effect to a certain extent. The impact may be related to the expression level of BCL-2, but it is unclear whether the mechanism is related to BCL-2 targeting and regulating the hippocampus of the brain. The apoptotic pathways in the region are related, and a mouse model needs to be established for further verification.

The body temperature rises during repeated febrile 
seizures, and the state of the brain is predominantly ischemic and hypoxic. The hippocampus is sensitive to this pathological state of ischemia and hypoxia, which can easily cause damage to the hippocampal cell structure. As early as 1985 , some scholars conducted research on rat models of febrile seizures and found significant changes in the structure of pyramidal cells in the hippocampus. Nucleus shrinkage, nucleolus densification, and vacuoles appeared in the cell body, which showed that the structure of nerve cells had been destroyed (17). The HE staining results of this study showed that the hippocampal pyramidal cells of normal young mice had normal morphology, while CFS mice showed different degrees of nucleus pyknosis, irregular cell morphology, and darker cytoplasmic staining. Similarly, after treatment with anti-epileptic drugs, the young rats displayed regular neuronal cells in the hippocampus, with a more uniform matrix, occasional pyknotic cells, and the lesions were less obvious than in the model group. The lesion score $(2.83 \pm 2.84)$ of the model group was significantly higher than that of the normal group $(0.83 \pm 1.33)$, VPA group $(0.92 \pm 1.31)$, and LEV group $(0.94 \pm 1.24)(\mathrm{P}<0.05)$, which verified that antiepileptic drugs can prevent or improve the damage the hippocampus from CFS. By measuring the level of BCL-2 in the hippocampus of mice, it was found that BCL-2 positive cells in the hippocampus of the normal group were rare, the hippocampus of the model group had more BCL-2 positive cells, and the positive cell staining and average optical density were enhanced. The hippocampal BCL-2 positive cells of the young rats in the LEV group decreased, and the positive cell staining and the average optical density also decreased. The average optical density was significantly lower than the model group $(\mathrm{P}<0.05)$. Combined with the results of the water maze test (the young mice in the VAP and LEV groups had lower learning and memory impairment), the reasoning that CFS can damage the hippocampus was further verified. Antiepileptic drugs can be used in the case of CFS to prevent this effect to a certain extent; the main mechanism is related to BCL-2 targeting and regulating apoptosis of the hippocampus.

\section{Conclusions}

In conclusion, antiepileptic drugs are effective in preventing the recurrence of CFS. The main mechanism may be related to BCL-2 targeting and regulating the apoptosis of hippocampal tissue in the nervous system. The onset and prognosis of CFS may be predicted by the use of BCL-2 as a potential early warning indicator.

\section{Acknowledgments}

Funding: This work was supported by Nantong Municipal Science and Technology Plan Guiding Project (JCZ19030).

\section{Footnote}

Reporting Checklist: The authors have completed the MDAR reporting checklist. Available at http://dx.doi.org/10.21037/ tp-20-458

Data Sharing Statement: Available at http://dx.doi. org/10.21037/tp-20-458

Conflicts of Interest: All authors have completed the ICMJE uniform disclosure form (available at http://dx.doi. org/10.21037/tp-20-458). The authors have no conflicts of interest to declare.

Ethical Statement: The authors are accountable for all aspects of the work in ensuring that questions related to the accuracy or integrity of any part of the work are appropriately investigated and resolved. All procedures performed in this study involving human participants were in accordance with the Declaration of Helsinki (as revised in 2013). The study was approved by Affiliated Hospital of Nantong University (NO. 2019005) and informed consent was taken from all the patients.

Open Access Statement: This is an Open Access article distributed in accordance with the Creative Commons Attribution-NonCommercial-NoDerivs 4.0 International License (CC BY-NC-ND 4.0), which permits the noncommercial replication and distribution of the article with the strict proviso that no changes or edits are made and the original work is properly cited (including links to both the formal publication through the relevant DOI and the license). See: https://creativecommons.org/licenses/by-nc-nd/4.0/.

\section{References}

1. Berg AT, Berkovic SF, Brodie MJ, et al. Revised terminology and concepts for organization of seizures and epilepsies: report of the ILAE Commission on Classification and Terminology, 2005-2009. Epilepsia 2010;51:676-85. 
2. Natsume J, Hamano SI, Iyoda K, et al. New guidelines for management of febrile seizures in Japan. Brain Dev 2017;39:2-9.

3. Martinos MM, Yoong M, Patil S, et al. Recognition memory is impaired in children after prolonged febrile seizures. Brain 2012;135:3153-64.

4. Rigo JM, Hans G, Nguyen L, et al. The anti-epileptic drug levetiracetam reverses the inhibition by negative allosteric modulators of neuronal GABA- and glycinegated currents. Br J Pharmacol 2002;136:659-72.

5. Lynch BA, Lambeng N, Nocka K, et al. The synaptic vesicle protein SV2A is the binding site for the antiepileptic drug levetiracetam. Proc Natl Acad Sci U S A 2004;101:9861-6.

6. Bea S, Zettl A, Wright G, et al. Diffuse large B-cell lymphoma subgroups have distinct genetic profiles that influence tumor biology and improve gene-expressionbased survival prediction. Blood 2005;106:3183-90.

7. Rentz DM, Weiss BK, Jacobs EG, et al. Sex differences in episodic memory in early midlife: impact of reproductive aging. Menopause 2017;24:400-8.

8. Morimoto T, Nagao H, Sano N, Takahashi M, Matsuda H. Electroencephalographic study of rat hyperthermic seizures. Epilepsia 1991;32:289-93.

9. Harding KL, Judah RD, Gant C. Outcome-based comparison of Ritalin versus food-supplement treated children with AD/HD. Altern Med Rev 2003;8:319-30.

10. Leung AK, Hon KL, Leung TN. Febrile seizures: an

Cite this article as: $\mathrm{Li} \mathrm{B,} \mathrm{Wu} \mathrm{Y,} \mathrm{He} \mathrm{Q,} \mathrm{Zhou} \mathrm{H,} \mathrm{Cai} \mathrm{J.} \mathrm{The}$ effect of complicated febrile convulsion on hippocampal function and its antiepileptic treatment significance. Transl Pediatr 2021;10(2):394-405. doi: 10.21037/tp-20-458 overview. Drugs Context 2018;7:212536.

11. Graves RC, Oehler K, Tingle LE. Febrile seizures: risks, evaluation, and prognosis. Am Fam Physician 2012;85:149-53.

12. Pavlidou E, Panteliadis C. Prognostic factors for subsequent epilepsy in children with febrile seizures. Epilepsia 2013;54:2101-7.

13. Steering Committee on Quality Improvement and Management, Subcommittee on Febrile Seizures American Academy of Pediatrics. Febrile seizures: clinical practice guideline for the long-term management of the child with simple febrile seizures. Pediatrics 2008;121:1281-6.

14. Natsume J, Hamano SI, Iyoda K, et al. New guidelines for management of febrile seizures in Japan. Brain Dev 2017;39:2-9.

15. Rantala H, Tarkka R, Uhari M. A meta-analytic review of the preventive treatment of recurrences of febrile seizures. J Pediatr 1997;131:922-5.

16. Hu LY, Zou LP, Zhong JM, et al. Febrile seizure recurrence reduced by intermittent oral levetiracetam. Ann Clin Transl Neurol 2014;1:171-9.

17. Chisholm J, Kellogg C, Franck JE. Developmental hyperthermic seizures alter adult hippocampal benzodiazepine binding and morphology. Epilepsia 1985;26:151-7.

(English Language Editor: J. Jones) 